Federal Reserve Bank of Minneapolis

Research Department Working Paper 619

March 2002

\title{
TECHNICAL APPENDIX: \\ Accounting for the Great Depression ${ }^{\dagger}$
}

\author{
V.V. CHARI \\ University of Minnesota \\ and Federal Reserve Bank of Minneapolis \\ PAtRick J. KehOE \\ Federal Reserve Bank of Minneapolis \\ and University of Minnesota \\ Ellen R. McGrattan \\ Federal Reserve Bank of Minneapolis \\ and University of Minnesota
}

$\dagger$ Codes and data are available upon request. The views expressed herein are those of the author and not necessarily those of the Federal Reserve Bank of Minneapolis or the Federal Reserve System. 
In this appendix we discuss the parameterization of the model and the computation of the equilibria. (See aea.f90/aea.inp.* for the Fortran code and input files used in computing the equilibria.) The model is slightly more general than that of the main text because we allow for time-varying distortions to labor that look either like labor income taxes or like consumption taxes, and we allow for time-varying distortions to capital that look either like capital income taxes or investment taxes. We also add growth and government spending to the economy.

\section{Computation of Model Equilibria Given the Wedges}

The consumers choose consumption per-capita $c_{t}$, per-capita investment $x_{t}$, and percapita labor $l_{t}$ to maximize

$$
\begin{array}{cl}
\max _{\left\{c_{t}, x_{t}, l_{t}\right\}} E \sum_{t=0}^{\infty} \beta^{t} U\left(c_{t}+\gamma g_{t}, 1-l_{t}\right) N_{t} \\
\text { subject to } & \left(1+\tau_{c t}\right) c_{t}+\left(1+\tau_{x t}\right) x_{t}=\left(1-\tau_{k t}\right) r_{t} k_{t}+\left(1-\tau_{l t}\right) w_{t} l_{t}+\tau_{k t} \delta k_{t}+T_{t} \\
& k_{t+1}=\left[(1-\delta) k_{t}+x_{t}\right] /\left(1+g_{n}\right) \\
& c_{t}, x_{t} \geq 0 \quad \text { in all states }
\end{array}
$$

where $k_{t}$ is the beginning-of-period per-capita capital stock, $g_{t}$ is per-capita government spending, $r_{t}$ is the rental rate on capital, $w_{t}$ is the wage rate, $\tau_{c t}$ is the distortion on consumption, $\tau_{x t}$ is the distortion on investment, $\tau_{k t}$ is the distortion on capital income, $\tau_{l t}$ is the distortion on labor income, and $T_{t}$ are per-capita transfers. We assume that the population grows at rate $g_{n}$ and technology grows at rate $g_{z}$. We will denote by $\hat{x}_{t}$ the detrended variable $x_{t} /\left(1+g_{z}\right)^{t}$.

For some of our parameterizations of the Great Depression, it is the case that the nonnegativity constraint on investment binds in some states. To deal with this possibility computationally, we add a penalty function to utility indexed by $\zeta$. The Lagrangian for 
the consumer's problem is thus

$$
\begin{aligned}
\mathcal{L}= & E \sum_{t} \tilde{\beta}^{t}\left\{U\left(\hat{c}_{t}+\gamma \hat{g}_{t}, 1-l_{t}\right)+\frac{\zeta}{3} \min \left(\hat{x}_{t}, 0\right)^{3}\right. \\
& +\mu_{t}\left\{\left(1-\tau_{k t}\right) r_{t} \hat{k}_{t}+\left(1-\tau_{l t}\right) \hat{w}_{t} l_{t}+\tau_{k t} \delta \hat{k}_{t}+\hat{T}_{t}-\left(1+\tau_{c t}\right) \hat{c}_{t}-\left(1+\tau_{x t}\right) \hat{x}_{t}\right\} \\
& \left.+\lambda_{t}\left\{(1-\delta) \hat{k}_{t}+\hat{x}_{t}-\left(1+g_{n}\right)\left(1+g_{z}\right) \hat{k}_{t+1}\right\}\right\}
\end{aligned}
$$

where $\tilde{\beta}=\beta\left(1+g_{n}\right) h\left(1+g_{z}\right)$ and $h(\cdot)$ depends on our choice of utility. If $U(c, 1-l)=$ $c^{1-\sigma} v(l)$, then $h\left(1+g_{z}\right)=\left(1+g_{z}\right)^{1-\sigma}$. Variables that grow over time are detrended (and are therefore given hats). Notice that the Lagrangian has no term for the nonnegativity constraint on investment. As $\zeta$ approaches infinity, the solution to the problem with a penalty function and no constraint on investment is the same as the solution to the original problem with $\zeta=0$ and $x_{t} \geq 0$ imposed. (See Fletcher (1987) for details.)

The first-order necessary conditions for the consumer problem are given as follows

$$
\begin{aligned}
& U_{1}\left(\hat{c}_{t}+\gamma \hat{g}_{t}, 1-l_{t}\right)=\mu_{t}\left(1+\tau_{c t}\right) \\
& U_{2}\left(\hat{c}_{t}+\gamma \hat{g}_{t}, 1-l_{t}\right)=\mu_{t}\left(1-\tau_{l t}\right) \hat{w}_{t} \\
& \zeta \min \left(\hat{x}_{t}, 0\right)^{2}+\lambda_{t}=\mu_{t}\left(1+\tau_{x t}\right) \\
& \left(1+g_{n}\right)\left(1+g_{z}\right) \lambda_{t}=\tilde{\beta} E_{t}\left[\lambda_{t+1}(1-\delta)+\mu_{t+1}\left\{\left(1-\tau_{k t+1}\right) r_{t+1}+\tau_{k t+1} \delta\right\}\right]
\end{aligned}
$$

along with the budget constraint and the constraint on capital accumulation.

We assume that firms have a technology given by $Y_{t}=F\left(K_{t}, Z_{t} L_{t}\right)$ which is homogeneous of degree 1 in $K$ and $Z L$ with $Z_{t}=z_{t}\left(1+g_{z}\right)^{t}$. Profit maximization implies that

$$
\begin{aligned}
r_{t} & =F_{k}\left(\hat{k}_{t}, z_{t} l_{t}\right) \\
\hat{w}_{t} & =F_{l}\left(\hat{k}_{t}, z_{t} l_{t}\right) z_{t} .
\end{aligned}
$$

If we eliminate the multipliers in (A1)-(A4) and substitute in factor prices, we have the following conditions:

$$
\frac{U_{2}\left(\hat{c}_{t}+\gamma \hat{g}_{t}, 1-l_{t}\right)}{U_{1}\left(\hat{c}_{t}+\gamma \hat{g}_{t}, 1-l_{t}\right)}=\frac{1-\tau_{l t}}{1+\tau_{c t}} F_{l}\left(\hat{k}_{t}, z_{t} l_{t}\right) z_{t}
$$




$$
\begin{gathered}
\frac{1+\tau_{x t}}{1+\tau_{c t}} U_{1}\left(\hat{c}_{t}+\gamma \hat{g}_{t}, 1-l_{t}\right)-\zeta \min \left(\hat{x}_{t}, 0\right)^{2} \\
=\hat{\beta} E_{t}\left[\frac{U_{1}\left(\hat{c}_{t+1}+\gamma \hat{g}_{t+1}, 1-l_{t+1}\right)}{1+\tau_{c t+1}}\right. \\
\left\{\left(1-\tau_{k t+1}\right) F_{k}\left(\hat{k}_{t}, z_{t} l_{t}\right)+\delta \tau_{k t+1}+(1-\delta)\left(1+\tau_{x t+1}\right)\right\} \\
\left.-(1-\delta) \zeta \min \left(\hat{x}_{t+1}, 0\right)^{2}\right]
\end{gathered}
$$

where $\hat{\beta}=\beta h\left(1+g_{z}\right) /\left(1+g_{z}\right)$. If $U(c, l)=c^{1-\sigma} v(l)$, then $\hat{\beta}=\beta\left(1+g_{z}\right)^{-\sigma}$. With the resource constraint,

$$
\hat{c}_{t}+\hat{g}_{t}+\hat{x}_{t}=\hat{y}_{t}=F\left(\hat{k}_{t}, z_{t} l_{t}\right)
$$

we have the equations we need to compute an equilibrium.

We now describe the exogenous processes for $\Gamma=\left\{\hat{g}, \tau_{k}, \tau_{l}, \tau_{c}, \tau_{x}, z\right\}$. Let $s$ index the state, where $s$ is determined by a $n$ th-order Markov chain. Then at time $t$, if the state is $s, \hat{g}_{t}=\hat{g}(s), \tau_{k t}=\tau_{k}(s), \tau_{l t}=\tau_{l}(s), \tau_{c t}=\tau_{c}(s), \tau_{x t}=\tau_{x}(s)$, and $z_{t}=z(s)$. The process for $s$ is intended to capture different stages of the depression. The transition matrix for $s$ is given by $\Pi$ with $\pi\left(s, s^{\prime}\right)$ being the probability of going from state $s$ to state $s^{\prime}$.

The state of the economy in any period can be summarized by two scalars: $\hat{k}$ and $s$. Our Fortran code in aea.f90 computes the decision rule $\hat{c}(\hat{k}, s)$. All other decisions can be determined via static first-order conditions once we know $\hat{c}(\hat{k}, s)$. In particular, $l(\hat{k}, s)$ is determined from (A5) and $\hat{x}(\hat{k}, s)$ is determined from (A7) once we know consumption.

To compute $\hat{c}(\hat{k}, s)$, we apply the Finite-Element method using the dynamic equation (A6) as the residual and Galerkin bases. (For an introduction to the Finite Element Method, see Reddy (1993).) More specifically, we assume that the consumption function is well approximated by

$$
\hat{c}(\hat{k}, s)=\sum_{j=1}^{\text {nnodes }} \alpha_{j}^{s} \Psi_{j}(k)
$$

where the $\Psi_{j}$ is a function that takes on nonzero values in 2 cells (or "elements") of a grid over $\hat{k}$ around grid point (or "node") $j$. 
The algorithm is to find the coefficients $\alpha_{j}^{s}, j=1, \ldots$ nnodes, $s=1, \ldots S$ that satisfy the following equations:

$$
\int R(\hat{k}, s ; \alpha) \Psi_{j}(\hat{k}) d \hat{k}=0
$$

for all $s$ and $j$ where

$$
\begin{aligned}
R(\hat{k}, s ; \alpha)= & \frac{1+\tau_{x}(s)}{1+\tau_{c}(s)} U_{1}(\hat{c}+\gamma \hat{g}(s), 1-l) \\
& -\zeta \min (\hat{x}, 0)^{2}+\hat{\beta}(1-\delta) \zeta \sum_{s^{\prime}} \pi_{s, s^{\prime}} \min \left(\hat{x}^{\prime}, 0\right)^{2} \\
& -\hat{\beta} \sum_{s^{\prime}} \pi_{s, s^{\prime}} \frac{U_{1}\left(\hat{c}^{\prime}+\gamma \hat{g}\left(s^{\prime}\right), 1-l^{\prime}\right)}{1+\tau_{c}\left(s^{\prime}\right)} \\
& \quad\left\{\left(1-\tau_{k}\left(s^{\prime}\right)\right) F_{1}\left(\hat{k}^{\prime}, z\left(s^{\prime}\right) l^{\prime}\right)+\tau_{k}\left(s^{\prime}\right) \delta+(1-\delta)\left(1+\tau_{x}(s)\right)\right\} .
\end{aligned}
$$

The investments $x$ and $x^{\prime}$ in (A8) satisfy resource constraints:

$$
\begin{aligned}
& \hat{x}=F(\hat{k}, z(s) l)-\sum_{j} \alpha_{j}^{s} \Psi_{j}(\hat{k})-\hat{g}(s) \\
& \hat{x}^{\prime}=F\left(\hat{k}^{\prime}, z\left(s^{\prime}\right) l^{\prime}\right)-\sum_{j} \alpha_{j}^{s} \Psi_{j}\left(\hat{k}^{\prime}\right)-\hat{g}\left(s^{\prime}\right) .
\end{aligned}
$$

The next period capital stock is given by:

$$
\hat{k}^{\prime}=((1-\delta) \hat{k}+\hat{x}) /\left[\left(1+g_{n}\right)\left(1+g_{z}\right)\right] .
$$

The labor inputs $l$ and $l^{\prime}$ solve:

$$
\begin{aligned}
& \frac{U_{2}(\hat{c}+\gamma \hat{g}(s), 1-l)}{U_{1}(\hat{c}+\gamma \hat{g}(s), 1-l)}=\frac{1-\tau_{l}(s)}{1+\tau_{c}(s)} F_{2}(\hat{k}, z(s) l) z(s) \\
& \frac{U_{2}\left(\hat{c}^{\prime}+\gamma \hat{g}\left(s^{\prime}\right), 1-l^{\prime}\right)}{U_{1}\left(\hat{c}^{\prime}+\gamma \hat{g}\left(s^{\prime}\right), 1-l^{\prime}\right)}=\frac{1-\tau_{l}\left(s^{\prime}\right)}{1+\tau_{c}\left(s^{\prime}\right)} F_{2}\left(\hat{k}^{\prime}, z\left(s^{\prime}\right) l^{\prime}\right) z\left(s^{\prime}\right) .
\end{aligned}
$$

\section{Computation of Wedges}

A Matlab file (wedges.m) was used to compute the efficiency and labor wedges that we use in our numerical experiments. For the results reported in the paper, we set $\tau_{c}$ and $\tau_{k}$ 
to constant values while $\tau_{l}, A=z^{1-\theta}$, and $\tau_{x}$ were time-varying. With these assumptions, we computed the wedges as follows:

$$
\begin{aligned}
& A_{t}=\hat{y}_{t} / F\left(\hat{k}_{t}, l_{t}\right) \\
& 1-\tau_{l t}=\left(1+\tau_{c}\right) \frac{U_{2}\left(\hat{c}_{t}+\gamma \hat{g}_{t}, 1-l_{t}\right)}{U_{1}\left(\hat{c}_{t}+\gamma \hat{g}_{t}, 1-l_{t}\right) A_{t} F_{l}\left(\hat{k}_{t}, l_{t}\right)} \\
& 1+\tau_{x t}=\left\{\left(1+\tau_{x t-1}\right) \frac{U_{1}\left(\hat{c}_{t-1}+\gamma \hat{g}_{t-1}, 1-l_{t-1}\right)}{\hat{\beta} U_{1}\left(\hat{c}_{t}+\gamma \hat{g}_{t}, 1-l_{t}\right)}-\left(1-\tau_{k}\right) A_{t} F_{k}\left(\hat{k}_{t}, l_{t}\right)-\tau_{k} \delta\right\} /(1-\delta)
\end{aligned}
$$

with $\hat{y}_{t}, \hat{k}_{t}, \hat{l}_{t}, \hat{c}_{t}$, and $\hat{g}_{t}$ from U.S. data, and $\hat{g}_{t}=\hat{y}_{t}-\hat{c}_{t}-\hat{x}_{t}$. We found that the last wedge, the investment wedge, implied a subsidy to investment. We do not use it in our numerical experiments since it will imply a boom rather than a depression. Later, we describe how we test theories with investment wedges.

The primary sources of data were the Bureau of Economic Analysis (BEA) National Income and Product Accounts (NIPA), Kendrick (1961), and Historical Statistics of the United States (1975). Most of the data is from NIPA. Manhours are taken from Kendrick (1961) Table A-X. For the results in the paper, we used civilian manhours. Population data are from Historical Statistics, Series A39. We used the population over 16.

The wedges.m code is written to allow us various assumptions about the NIPA accounts and the assignment of GNP components to variables in the model. In particular, we allowed for the following variations

1. Durables could be included with investment or private consumption;

2. Net foreign investment could be included or excluded from the accounts;

3. Government investment could be included with government consumption $(g)$ or with private investment $(x)$ or split between $g$ and $x$ with military included in $g$ and the remainder in $x$;

4. Owner-occupied housing could be included or excluded from the accounts.

When simulating the results in the paper, we assumed that durables were included 
Table 1. Parameter Choices

\begin{tabular}{|l|l|l|}
\hline Growth in population & $g_{n}$ & $1.5 \%$ \\
Growth in technology & $g_{z}$ & $1.6 \%$ \\
Depreciation rate & $\delta$ & $6 \%$ \\
Capital share & $\theta$ & $34 \%$ \\
Consumption distortion & $\tau_{c}$ & $3.7 \%$ \\
Investment distortion & $\tau_{x}$ & $0 \%$ \\
Capital distortion & $\tau_{k}$ & $30 \%$ \\
Labor distortion & $\tau_{l}$ & $3.5 \%$ \\
Discount factor & $\beta$ & 0.97 \\
Utility leisure weight & $\psi$ & 2.26 \\
Government consumption share & $g / y$ & $7.6 \%$ \\
\hline
\end{tabular}

with investment, net foreign investment was excluded from the accounts, government investment was split with military in government consumption and the remainder in private investment, and owner-occupied housing excluded from the accounts. We tried variations on these assumptions but got very similar results so we only reported one case.

\section{Functional Forms and Parameters}

We assume the following

$$
\begin{aligned}
& u(c, 1-l)=\left[c(1-l)^{\psi}\right]^{\phi} / \phi \\
& F(k, l)=k^{\theta} l^{1-\theta} .
\end{aligned}
$$

For the results in the paper, we assumed $\log$ utility $(\phi=0)$. The rest of the parameters were chosen to be consistent with pre-depression levels of growth, consumption, investment, capital, hours, capital share, and tax rates. (For pre-depression data, see Romer (1989) and Kendrick (1961).) Thus, if there are no changes in wedges, the model delivers a balanced growth path in which levels are consistent with observations in the late 1920s. Table 1 reports the specific values we used.

For the results in the paper, the expectations were modeled as follows. In the first three years following the start of the depression, we assumed that each of the following 
three events is equally likely: the wedges revert to their values at the beginning of the depression, the wedges stay at their current value and the wedges take on the values in the subsequent year. Following these three years we assume perfect foresight until the end of the depression, in the sense that agents foresee the actual values the wedges take on. At the end of the depression we assume that the labor wedge is constant at its last period value and the technology parameter grows from its last period value at the balanced growth rate.

\section{The Experiments}

The numerical experiments were reported in Figures 2-4. Figure 2 shows two simulations of the model economy - with only one wedge on at a time. The input files for these cases are aea.inp.a and aea.inp.taun. For these simulations we set the other exogenous inputs in $\Gamma$ at their 1929 levels.

Figure 3 shows simulations with both the efficiency and labor wedges from data input into the model. The input file for this case is aea.inp.ataun. Here, we also allow government spending to be what it was in the data. Since there is little variation of this during the

depression, including or excluding variation in government spending does not have much affect on the figures.

Finally, the results of Figure 4 are generated as follows: we choose the variation in $\tau_{x}$ to be whatever it has to be to have investment in the model equal to investment in the data. All other variables in $\Gamma$ are assumed to be constant at their 1929 levels. Parameters and expectations are the same as in the cases of Figures 2 and 3. The input file for this case is aea.inp.taux. 


\section{References}

Fletcher, R. 1987. Practical Methods of Optimization. New York: Wiley \& Sons.

Kendrick, John W. 1961. Productivity Trends in the United States. Princeton, NJ: Princeton University Press.

Reddy, J.N. 1993. An Introduction to the Finite Element Method. New York: McGrawHill.

Romer, C.D. 1989. The prewar business cycle reconsidered: New estimates of gross national product, 1869-1908. Journal of Political Economy 97:1-37.

U.S. Bureau of Economic Analysis. 2002. National Income and Product Accounts. http://www.bea.doc.gov.

U.S. Bureau of the Census. 1975. Historical Statistics of the United States, Colonial times to 1970. Bicentennial edition. Washington, D.C. 